\title{
Meta
}

Journal des traducteurs

Translators' Journal

\section{L'enseignement des langues de spécialité comme préparation à la traduction spécialisée}

\section{Jacques Lethuillier}

Volume 48, numéro 3, septembre 2003

Traduction et enseignement

Translation and teaching

URI : https://id.erudit.org/iderudit/007598ar

DOI : https://doi.org/10.7202/007598ar

Aller au sommaire du numéro

Éditeur(s)

Les Presses de l'Université de Montréal

ISSN

0026-0452 (imprimé)

1492-1421 (numérique)

Découvrir la revue

Citer cet article

Lethuillier, J. (2003). L’enseignement des langues de spécialité comme préparation à la traduction spécialisée. Meta, 48(3), 379-392.

https://doi.org/10.7202/007598ar
Résumé de l'article

Dans la formation des traducteurs professionnels modernes, on retrouve presque à égalité deux grandes composantes : la traduction générale et l'initiation à la traduction spécialisée. Si l'on peut dire que l'enseignement de la traduction générale repose sur une tradition bien établie, rien n'est moins vrai en ce qui concerne l'initiation à la traduction spécialisée. Dans ce texte, nous défendons l'idée que la meilleure façon de préparer à la traduction spécialisée consiste à dispenser un enseignement des langues de spécialité systématique, qui se fonde sur une pédagogie originale dont nous essayons de tracer les grandes lignes.
Ce document est protégé par la loi sur le droit d'auteur. L'utilisation des services d’Érudit (y compris la reproduction) est assujettie à sa politique d'utilisation que vous pouvez consulter en ligne.

https://apropos.erudit.org/fr/usagers/politique-dutilisation/ 


\title{
L'enseignement des langues de spécialité comme préparation à la traduction spécialisée
}

\author{
JACQUES LETHUILLIER \\ Université de Montréal, Montréal, Canada \\ jacques.lethuillier@umontreal.ca
}

\begin{abstract}
RÉSUMÉ
Dans la formation des traducteurs professionnels modernes, on retrouve presque à égalité deux grandes composantes: la traduction générale et l'initiation à la traduction spécialisée. Si l'on peut dire que l'enseignement de la traduction générale repose sur une tradition bien établie, rien n'est moins vrai en ce qui concerne l'initiation à la traduction spécialisée. Dans ce texte, nous défendons l'idée que la meilleure façon de préparer à la traduction spécialisée consiste à dispenser un enseignement des langues de spécialité systématique, qui se fonde sur une pédagogie originale dont nous essayons de tracer les grandes lignes.
\end{abstract}

\section{ABSTRACT}

Today translator training consists of two main components of equal importance: general translation and introduction to specialized translation. If there is a well established tradition for teaching general translation, the same cannot be said for specialized translation. In this article, the author suggests that the best preparation for specialized translation is systematic teaching of specialized language, and outlines an original framework for such teaching.

\section{MOTS-CLÉS/KEYWORDS}

enseignement de la traduction, formation, langues de spécialité, traduction spécialisée

Traditionnellement, la formation des traducteurs professionnels se trouve centrée sur l'acquisition d'une compétence en traduction générale. On sait que cette aptitude à la traduction générale met en jeu principalement une connaissance et un savoir-faire. La connaissance est celle de la langue de départ et celle de la langue d'arrivée. Le savoir-faire est l'aptitude à décoder les textes écrits dans une langue pour en projeter le sens dans une autre langue, sous la forme d'un texte «équivalent». Or, contrairement à ce qu'on pourrait penser, l'expérience montre que très peu de gens possèdent naturellement ce savoir-faire. Cela explique que son acquisition occupe une place prépondérante dans les programmes de formation des traducteurs professionnels. En outre, la connaissance du couple de langues que vise la formation est considérée comme un préalable.

Depuis de nombreuses années maintenant, la traduction des textes spécialisés est devenue une activité importante partout à travers le monde. Dans la compétence du traducteur professionnel, l'aptitude à exécuter ce type de traduction constitue désormais une composante essentielle. Ainsi, la compétence du traducteur professionnel moderne ne saurait se limiter à la traduction générale. Le futur traducteur professionnel doit se préparer à la traduction spécialisée. Comment? Répondre à cette question 
n'est pas aisé. Une façon simple d'aborder le problème consiste à s'interroger sur ce qui fait la différence entre la traduction générale et la traduction spécialisée.

\section{Le processus de traduction}

FIGURE 1

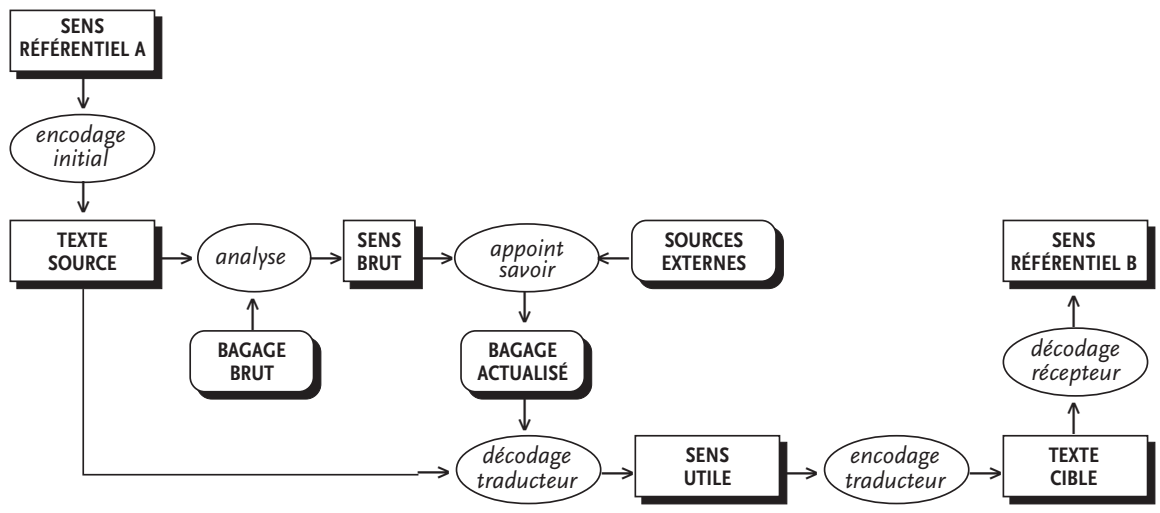

À cette fin, il est pratique de revenir sur le processus de traduction en général, qu'illustre la figure 1. On voit qu'il s'inscrit dans une chaîne de communication qui part du vouloir dire de l'auteur (sens référentiel $\mathrm{A}$ ) et aboutit à l'interprétation du destinataire (sens référentiel B). Nous évoquons sur ce schéma la capacité du traducteur à dégager un «sens utile» à partir du texte source et à le projeter dans la langue d'arrivée sous la forme d'un texte cible possédant les qualités voulues pour que le destinataire en dégage un sens aussi proche que possible du vouloir dire de l'auteur. Idéalement, le sens référentiel $\mathrm{B}$ se confondrait avec le sens référentiel A.

Ce schéma, avec sa boucle représentant la phase de préparation, à savoir la phase d'appoint de connaissances, décrit particulièrement bien l'activité du traducteur quand il se voit confier la tâche de traduire des textes pragmatiques spécialisés. En résumé, on y distingue nettement trois phases:

- préparation ou appoint de connaissances;

- décodage (dégagement d'un sens utile à partir du texte $\mathrm{LD}$ );

- encodage (projection du sens utile sous la forme d'un texte en LA adapté).

Le concept de traduction générale, c'est un peu l'idée que la phase de préparation est réduite à sa plus simple expression, que le processus de traduction n'exige pas d'appoint de connaissances. Ce serait aussi, par voie de conséquence, le fait que le sens utile se confond avec les sens référentiels A et B.

À l'inverse, on parle de traduction spécialisée dès que le bagage cognitif partagé par le plus grand nombre ne suffit pas pour mener à bien les opérations de décodage et de transcodage, qu'un appoint de connaissances s'impose. Ainsi, dans le processus de la traduction spécialisée, l'appoint de connaissances revêt un caractère essentiel. Ce point amène à s'interroger sur la compréhension utile du texte à laquelle doit 
parvenir le traducteur. Et de là à se demander s'il est possible de préciser clairement la nature des connaissances nécessaires pour parvenir à cette compréhension, puis à se demander si ce type de connaissance doit faire l'objet d'un enseignement, et sous quelle forme alors, dans l'affirmative.

\section{Langues de spécialité: divers obstacles à surmonter}

Avant d'aller plus loin, il importe d'essayer de préciser en quoi consistent ces connaissances utiles pour le traducteur, notamment par rapport au savoir du spécialiste. Elles représentent moins que le savoir du spécialiste, et plus à la fois. Moins, parce que ces connaissances utiles sont essentiellement la connaissance des moyens d'expression du spécialiste, autrement dit de la langue de spécialité utilisée par ce dernier. Et plus, parce que cette connaissance des moyens d'expression des spécialistes doit être acquise dans une perspective bilingue, comparatiste.

Par ailleurs, il est indispensable d'apprécier les paramètres de la situation d'apprentissage, et en particulier, de prendre la mesure des obstacles à surmonter pour élaborer un enseignement qui conduise à l'appropriation efficace des connaissances spécialisées que représente une langue de spécialité, dans une perspective bilingue, comparatiste, compte tenu du profil de profane qui caractérise les apprenants.

Ces obstacles concernent d'abord les systèmes constitués par les langues de spécialité. On peut s'effrayer de leur énormité, du rythme de leur prolifération, de la complexité de leur fonctionnement, des défauts de régularité qui les caractérisent et de leur nature rapidement évolutive. Ils concernent ensuite les déficits observables dans leurs descriptions, en particulier dans les descriptions des systèmes notionnels, à voir comme le noyau dur des langues de spécialité. Ces déficits touchent à la fois la macrostructure et la microstructure des outils que sont les dictionnaires spécialisés. Les descriptions terminographiques recèlent des lacunes thématiques et en même temps des lacunes dans le traitement. Et le constat pessimiste qui vaut pour ces outils de description en extension des langues de spécialité s'applique tout autant à leur description en compréhension, c'est-à-dire à la description de leur fonctionnement ou de leur structure.

Devant un tel tableau, il serait normal de mal réagir et de rejeter la possibilité d'opter pour une préparation à la traduction spécialisée par un enseignement méthodique des langues de spécialité. À moins de contourner les principaux obstacles grâce à une approche particulièrement adaptée: une approche qui contienne en puissance les réponses aux obstacles apparaissant les plus insurmontables; une approche conduisant à une optimisation de la démarche d'apprentissage par le recours à des moyens du type levier, dont les effets se cumuleraient et se renforceraient. Il faut trouver des réponses au défi de la complexité et à celui de l'énormité, qui représentent les deux paramètres principaux de la situation d'apprentissage. 
FIGURE 2

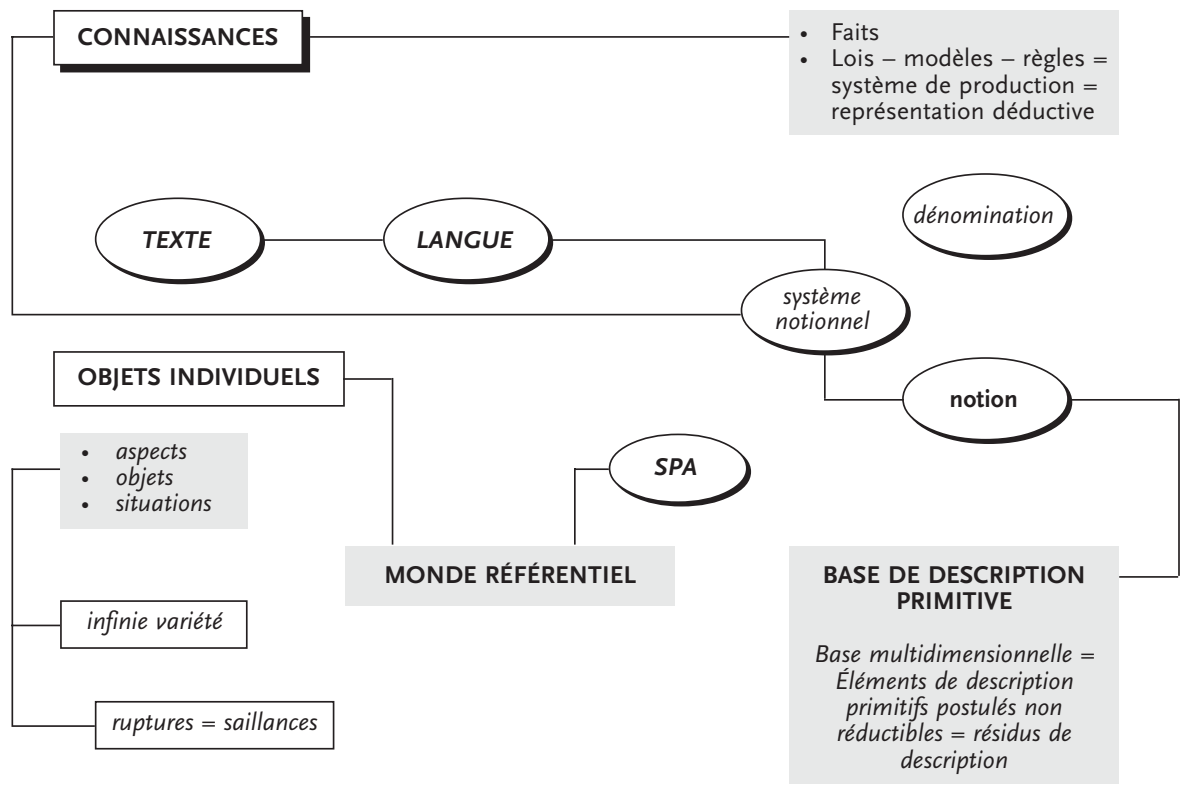

Une réponse au défi de l'énormité serait d'amener les étudiants à l'autonomie dans l'apprentissage. Et une partie de la réponse au défi de la complexité est à chercher dans une métaconnaissance de la connaissance et des moyens de l'exprimer. On ne saurait en effet parler d'appoint de connaissances sans essayer de cerner l'idée de connaissance, sans s'interroger sur les diverses formes de représentation des connaissances, sans évoquer le rôle prépondérant joué par la ou les langues dans la représentation des connaissances. Ni encore sans évoquer la centralité du lexique dans cette fonction de la langue.

\section{Définir la connaissance: \\ de la réalité extralinguistique à la Langue}

"Les connaissances, c'est ce que l'on a appris", nous dit le Grand dictionnaire encyclopédique Larousse. Sa définition peut nous laisser perplexe. En tout cas, elle ne nous apprend pas grand-chose. Il n'est pas question pour nous de théoriser à propos de l'idée de connaissance. Toutefois, avant de parler concrètement d'une manière particulière d'acquérir des connaissances dans un contexte lui-même particulier, il nous paraît utile de préciser les liens qui existent entre la notion de connaissance et un certain nombre de notions fortement imbriquées avec cette dernière. Nous pensons notamment à la réalité extralinguistique, à la langue et à d'autres notions comme celle d'objet individuel, dont les contours sémantiques sont passablement flous, sans doute en raison de leur caractère primitif ou quasipostulé.

La figure 2 nous amène à voir la connaissance comme un produit et rappelle qu'elle est ce que l'on sait sur la réalité extralinguistique. Notre schéma représente une chaîne ouverte reliant deux pôles ou nœuds terminaux. La chaîne part de la 
réalité extralinguistique, que l'on peut encore qualifier de monde ou d'univers référentiel, et elle aboutit à la connaissance, produit d'une interaction entre cette réalité extralinguistique et le système de perception et d'analyse humain (SPA, sur notre schéma). Cette interaction est un processus, une forme de traitement.

La réalité extralinguistique englobe tout ce qui est accessible à notre système de perception et d'analyse, sans aucune limite. Un élément de cette réalité est isolable par notre système de perception et d'analyse, et l'on peut y référer. Il constitue un objet particulier ou individuel. On dit encore un référent, de sorte que la réalité extralinguistique est parfois qualifiée de monde référentiel. L'étiquette «objet individuel» n'est d'aucune façon réservée. Tout est potentiellement objet individuel, aussi bien le tout que la partie, les situations comme les éléments qui les composent. Les aspects nous servant à les décrire sont également objets individuels, si on veut les voir ainsi. Les objets individuels se distribuent entre les univers concrets et les univers abstraits que représentent les constructions mentales.

Que dire maintenant du deuxième pôle de notre schéma, le nœud terminal représentant la notion de connaissance? Nous avons vu que l'idée de connaissance ne peut s'aborder sans évoquer celle de réalité extralinguistique et d'objet individuel, dont on est obligé de reconnaître la prééminence. Le lien entre les deux est grossièrement traduit par l'affirmation que les connaissances forment une description de la réalité extralinguistique. Nous pourrions ajouter que la connaissance ou un système de connaissances est un produit mental élaboré à partir du monde référentiel. En paraphrasant, cela devient: la connaissance est le résultat d'une interaction de notre système de perception et d'analyse avec le monde référentiel. C'est une construction mentale qui forme dans notre esprit une réplique du monde référentiel.

Deux questions se posent. Tout d'abord, quelle est en gros la nature de cette construction? Nous parlons de résultat, de produit. Idéalement, la connaissance, c'est la description complète de tous les objets individuels d'un monde référentiel ou de mondes référentiels, et en particulier l'explicitation de tous les liens existant entre eux. En bref, la connaissance pourrait être un gigantesque catalogue de faits singuliers. Compte tenu de l'infinie variété des objets individuels, la connaissance ou la description du monde référentiel serait impossible à encoder et à utiliser sous une telle forme. Au lieu de cela, les connaissances sur le monde référentiel présentent un caractère déductif. Ce sont donc plutôt des systèmes de production, réunissant des règles, des lois ou des modèles, qui nous permettent, à partir de certains faits singuliers avérés, de déduire de nouveaux faits.

En outre, toutes les formes de représentation des connaissances sont fondées sur la Langue. On utilise en particulier la fonction de catégorisation de celle-ci, c'est-àdire les systèmes notionnels, qui permettent de tenir des propos généralisant, de dire des choses vraies non pas sur des objets individuels mais sur des ensembles d'objets individuels, réunis en classes terminologiques. Ainsi, la Langue, et plus particulièrement le lexique, est au cœur des connaissances. Le groupement des objets individuels en classes représente une étape dans le processus d'élaboration des connaissances, et les systèmes notionnels sont le substrat sur lequel s'édifie tout système de connaissances. 


\section{Constructions mentales et lexique: quelques questions fondamentales}

On sait que les systèmes notionnels sont des collections de notions apparentées. Ces collections se trouvent répertoriées sous forme discursive dans des catalogues que l'on appelle des dictionnaires. Les notions ne sont pas autre chose que des schémas de description d'objets individuels discriminants, qui jouent le rôle de filtre référentiel, c'est-à-dire réalisent le groupement utile d'objets individuels en ensembles appelés classes terminologiques. Ces schémas de description forment des outils de description puissants en même temps qu'ils offrent un moyen de désignation collective des objets individuels.

Le lexique est à la fois une connaissance, la base ou le point de départ de tout processus d'élaboration de connaissances et en même temps la composante centrale de tout formalisme de représentation des connaissances. Pour se convaincre de ce dernier point, il suffit de penser à la représentation mathématique des connaissances. Le lexique est en quelque sorte le produit de base de l'interaction de notre système de perception et d'analyse avec le monde référentiel, qui conduit à élaborer une réplique de ce dernier dans notre esprit sous la forme de constructions mentales représentant un découpage utile de la réalité.

À propos des systèmes notionnels, nous avons parlé de construction mentale, et la métaphore n'est pas si mauvaise. Évoquant l'idée de construction, on peut se demander à partir de quels matériaux est bâti l'édifice que représente le lexique. De quoi sont faites les notions. Nous avons dit que les notions ne sont pas autre chose que des schémas de description, autrement dit des descriptions partielles d'objets individuels, et il est raisonnable de postuler que ces schémas de description sont faits à partir de constituants élémentaires formant un alphabet de description. Un tel alphabet est à voir comme un produit hypothétique, sur lequel se fonderait implicitement la construction du lexique.

L'idée d'alphabet de description postulé renvoie à la notion classique de primitive, facile à faire comprendre. Il suffit de penser à ce qu'est un dictionnaire de définitions. Chaque entrée ou forme linguistique servant d'étiquette à une notion est suivie d'un énoncé définitoire qui est assimilable à un prédicat complexe. Si, dans chaque énoncé définitoire, on remplaçait par leur définition les termes y figurant et faisant l'objet d'une entrée dans le dictionnaire, on obtiendrait évidemment sous chaque entrée du dictionnaire des textes définitoires très longs, mais qui seraient composés à partir d'un nombre limité d'éléments descripteurs fondamentaux. Cela ferait "sortir » notre alphabet de description hypothétique, qui réunirait des prédicats simples, assimilables à des résidus de description, dans la mesure où il s'agit de sens que l'on postule sans avoir la possibilité de les décrire. Il s'agit d'atomes conceptuels, autrement dit d'éléments de description indécomposables des objets individuels ou, si l'on préfère, non définissables à partir d'éléments de description qui seraient plus simples. Et peu importe que ce ne soit pas possible de recenser ces résidus de description. L'important est de postuler leur existence, de postuler qu'ils forment une base à partir de laquelle tous les sens complexes peuvent se décrire, une base dans laquelle ils peuvent être représentés.

Notre effort pour décrire globalement les langues de spécialité est resté au niveau des généralités. Le lexique ou les systèmes notionnels font tellement partie de nous 
que l'on se pose rarement des questions à leur sujet. Certaines questions fondamentales ne nous effleurent pour ainsi dire jamais l'esprit. Quels critères utilise la Langue pour donner à des schémas de description le statut de notion terminologique? Cette question nous renvoie à l'idée de groupement utile. Quelle est la structure des notions? Les notions ne sont pas des agrégats de caractères, comme on le lit trop souvent, dans la mesure où cela laisse entendre qu'aucun ordre n'existe derrière les éléments de description irréductibles qui les composent. Que peut-on dire des liens entre les notions et les formes linguistiques utilisées pour y référer, autrement dit pour les manipuler dans le processus de communication? Il s'agit là de tout un discours essentiel sur la motivation, dont on oublie en général que c'est une clé du fonctionnement des systèmes linguistiques. Enfin, quelle est la structure des systèmes notionnels?

\section{Appropriation des langues de spécialité: la sélection thématique}

La connaissance abstraite du fonctionnement des langues de spécialité peut servir de levier pour leur apprentissage, jusqu'à un certain point. Par ailleurs, il est sans doute rassurant de penser que le traducteur spécialisé n'a pas besoin, pour remplir sa fonction efficacement, de posséder les connaissances du spécialiste, que seulement une partie de ses connaissances, celles se rapportant aux moyens d'expression utilisés par ce dernier (autrement dit les langues de spécialité), lui sont nécessaires. Néanmoins, en dépit du fait que le bagage représenté par la connaissance des langues de spécialité est relativement limité au regard du savoir des spécialistes, son appropriation, même partielle, représente un véritable défi en raison de son énormité et de sa complexité.

Dans la réponse au défi de l'énormité intervient certainement la métaconnaissance, mais cette dernière est plus utile pour définir la forme et le contenu de l'enseignement que pour faciliter l'apprentissage. En fait, la vraie réponse au défi de l'énormité et du foisonnement des langues de spécialité s'articule autour d'un objectif double, complémentaire de l'objectif d'autonomie dans l'apprentissage évoqué précédemment. Il s'agit de bien cibler les connaissances utiles, d'une part, et de maximiser le taux de rétention, d'autre part.

«Bien cibler» relève d'une stratégie thématique. Appliquer une stratégie thématique est une opération de balisage visant à assurer la portée de l'apprentissage, à assurer que le traducteur en formation s'approprie des ressources utiles pour remplir efficacement sa fonction. La stratégie thématique concerne en premier lieu l'appréciation de la pertinence des thèmes pour le traducteur, la pertinence de connaître les langues de spécialité s'y rapportant, d'en faire l'apprentissage. De ce point de vue, les langues de spécialité ne sont pas égales. Il existe des langues de spécialité plus universelles que d'autres, plus centrales que d'autres, et aussi plus accessibles que d'autres. Les critères de choix sont nombreux, complexes et évolutifs.

La sélection thématique ne vise pas seulement le choix logique, pour utiliser un qualificatif très global, des thèmes à retenir et à explorer. Il y a aussi un balisage à faire à l'intérieur des thèmes. Ce balisage interne est plus strict, les critères qui le fondent s'imposant avec plus de netteté. Plutôt que de sélection, on pourrait parler de centrage, même s'il s'agit toujours d'élaguer. L'important est de faire porter l'effort d'apprentissage principalement sur la partie centrale des systèmes notionnels (notions 
génériques et spécifiques prototypiques), sur la cooccurrence et sur ce que nous appelons les phénomènes de brouillage linguistique et interlinguistique (interférences des deux langues visées, de la langue générale et des langues de spécialité, polysémie et synonymie complexe, etc.).

On a évoqué plus tôt un premier objectif de formation global: rendre l'étudiant autonome dans l'apprentissage. Par ailleurs, après avoir fait état de l'intérêt, comme moyen d'appréhender la complexité de tels systèmes, d'orienter la formation vers l'acquisition d'une connaissance abstraite des langues de spécialité, il a été question de critères à appliquer pour centrer et baliser l'apprentissage. L'idée est de se donner la capacité de distinguer entre l'essentiel et le détail, de ne pas se perdre dans l'immensité des systèmes représentés par les langues de spécialité et de garder à l'esprit la perspective dans laquelle doit se faire leur appropriation.

\section{L'étape concrète d'apprentissage}

C'est comme si nous avions fixé un cadre d'exploration des langues de spécialité, où pourrait s'installer une démarche optimale pour leur apprentissage dans une perspective bilingue, comparatiste, particulièrement adaptée aux besoins du traducteur. En gros, cela revenait à préciser ce que l'on ciblait. Vient ensuite seulement l'étape concrète d'apprentissage. On en arrive au «comment s'approprier le plus efficacement possible le type de connaissances ciblées» ou «comment concevoir un enseignement allant dans le même sens».

La démarche s'organise en fonction de la nature des connaissances à acquérir. À ce sujet, il convient d'opposer les connaissances notionnelles aux connaissances davantage centrées sur la langue, à voir comme l'objectif principal de l'exploration. Les connaissances notionnelles sont un prétexte. Néanmoins, en raison de leur forte imbrication des unes dans les autres, l'appropriation des connaissances centrées sur la langue ne saurait se faire convenablement tant qu'un bagage cognitif minimal n'a pas été acquis. Ainsi, la démarche d'exploration met en jeu une composante notionnelle formant un substrat sur lequel se greffe la composante spécifiquement langagière, plus proche de l'objectif principal d'apprentissage. À partir de points précis de l'exploration quasilinéaire d'un système notionnel, sont menées des excursions langagières qui, à l'occasion, feront sortir du thème exploré.

Il s'agit là de la structure générale caractérisant la démarche. Dans le détail, comment parvient-on à maximiser le taux de rétention et les retombées de l'exploration, notre deuxième objectif? Il convient d'abord de respecter une série de principes pour bien mener l'exploration notionnelle. Par exemple: exploiter l'effet pédagogique de la répétition; atteindre une certaine "masse critique», pour ne pas papillonner; aller du concret à l'abstrait, du simple au complexe et du connu vers le nouveau; et enfin, multiplier les ancrages des notions en visualisant le plus grand nombre de liens possible entre elles. On parvient à respecter ces principes en recourant au levier que constituent les groupements thématiques et en utilisant bien ces derniers, notamment pour définir un ordre de présentation des notions favorisant leur appropriation, en d'autres termes un fil directeur optimal pour l'exploration notionnelle. 


\section{Les groupements thématiques}

Qu'entendons-nous par groupement thématique? Un groupement thématique est une sorte de réseau sémantique où l'on représente graphiquement des ensembles de notions relatives à un thème que l'on relie par des lignes matérialisant le rapport qui les unit. En général, les lignes ne sont pas étiquetées, alors que les notions, appelées nœuds, le sont par leur nom. Le plus souvent, les groupements thématiques réunissent des notions apparentées qui entretiennent des liens ou rapports d'un même type entre elles. On parle alors de groupements homogènes. À l'occasion, on groupera des notions qui sont liées les unes aux autres par des rapports variés. Il serait question de groupement pragmatique. Le choix des notions repose quand même sur un critère, mais ce critère n'est pas simple.

Deux grandes classes de groupements thématiques de type homogène sont à distinguer: les groupements logiques réunissant des notions entre lesquelles existe un rapport de générique à spécifique et les groupements ontologiques réunissant des notions entre lesquelles existe un rapport de nature réelle, toujours du même type.

Les groupements logiques, qui sont particulièrement utiles, se présentent comme des arborescences. Leur exploration permet d'exploiter efficacement la propriété d'héritage du contenu sémantique des génériques par les spécifiques. Ils représentent une aide précieuse quand il s'agit de faire des descriptions sous forme discursive des systèmes notionnels. La navigation verticale dans ces structures, du concret (niveau référentiel) à l'abstrait, ou l'inverse, facilite grandement l'appropriation des notions. La navigation horizontale a le même genre d'effet. Elle permet souvent de s'appuyer sur des spécifiques prototypiques connus et sur un système d'opposition pour consolider l'apprentissage des notions génériques.

À côté des groupements thématiques qui font ressortir des rapports logiques, il est très utile de s'appuyer sur des groupements de notions, toujours thématiques (on ne sort pas du thème), mettant en relief des relations ontologiques (rapports sémantiques réels existant entre les notions). Ces groupements thématiques homogènes peuvent se faire en fonction de rapports variés (tout-partie, matière-produit, succession dans le temps, succession dans l'espace, cause-effet, etc.). Dans la pratique, le type de groupement le plus productif à notre fin est basé sur le rapport de tout à partie.

Les groupements thématiques ne sont pas seulement un moyen de visualiser des liens entre des notions apparentées ressortissant à un thème dans le but de faciliter leur appropriation. Ils représentent aussi un outil pour l'établissement d'un ordre d'exploration. Le recours simultané, en alternance, à des structures générique-spécifique et à des structures ontologiques (notamment tout-partie) peut se révéler très efficace pour définir un fil directeur d'exploration, c'est-à-dire un ordre de présentation ou d'appropriation des notions relativement à un thème. Le fil directeur, c'est d'abord l'ordre des plans ou groupements thématiques, généralement linéaire, mais pouvant comporter des retours en arrière, qui correspond à une chaîne ouverte, l'exploration se déroulant depuis un plan initial jusqu'à un plan final. Et c'est aussi l'ordre selon lequel se fait la navigation entre les nouds (notions) à l'intérieur de chaque plan. Ces ordres répondent à une logique pédagogique, respectant certains principes évoqués ci-dessus, et leur ensemble définit un fil directeur.

Ainsi, concrètement, établir un fil directeur revient à définir une succession ordonnée de plans ou de réseaux notionnels se présentant le plus souvent sous forme 
d'arborescences, qui réunit aussi bien des structures logiques (réseaux abstraits) que des structures ontologiques (réseaux concrets), l'ordre d'exploration des plans pouvant comporter des retours en arrière. Établir un fil directeur, c'est aussi définir un ordre d'exploration des nœuds à l'intérieur de chaque plan et préciser à partir de certains nœuds quand sortir d'un plan pour effectuer des excursions à caractère linguistique.

FIGURE 3

Exemple d'un thème: les pompes et les moteurs
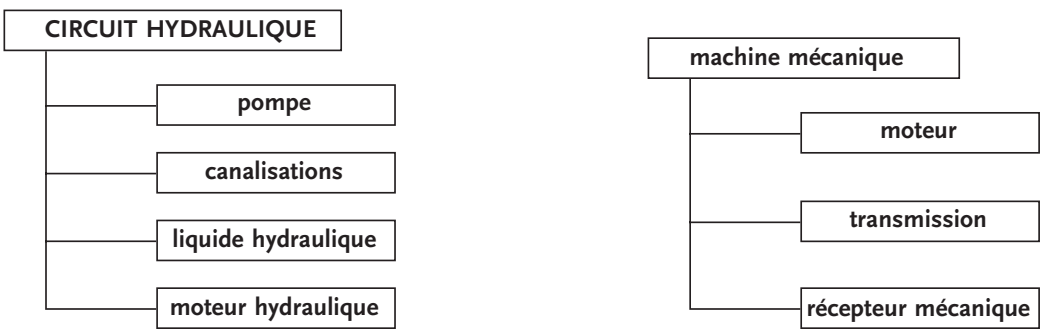

Si le thème à explorer était les pompes ou les moteurs, on inscrirait d'abord les notions de pompe ou de moteur dans une structure tout-partie (voir figure 3 ), où l'environnement réel le plus courant de ces types d'objets individuels serait présenté. La figure 3 nous dit que l'environnement le plus courant correspondant à l'utilisation des pompes est leur insertion dans un circuit hydraulique. La lecture de cette figure conduit à une première appropriation de la notion d'un point de vue très concret. En gros, la lecture à faire de l'arborescence ci-dessus serait la suivante. Les objets individuels du type pompe sont des générateurs d'énergie hydraulique en même temps que des récepteurs mécaniques. Ils communiquent l'énergie hydraulique qu'ils produisent à partir d'énergie mécanique par l'intermédiaire d'un liquide hydraulique circulant dans une canalisation, à un récepteur (hydraulique) ne pouvant guère prendre d'autre forme que celle de moteur hydraulique, et il y a retour. L'ensemble pompe, canalisation, liquide hydraulique et moteur hydraulique constitue l'essentiel d'un circuit hydraulique.

La figure 3 établit un parallèle entre la notion de machine mécanique et celle de circuit hydraulique. Elle fait ressortir les principaux composants de toute machine mécanique, à savoir: un moteur ou une machine motrice, un récepteur mécanique ou une machine réceptrice et un mécanisme de transmission ou, plus simplement, une transmission. Une telle arborescence ne met pas en évidence que ces composants forment une chaîne, ouverte dans ce cas, ni l'ordre de succession de ces composants par rapport au mouvement transmis.

Une autre figure simple pourrait le faire sur le cas particulier d'une machine de locomotion terrestre. 
FIGURE 4

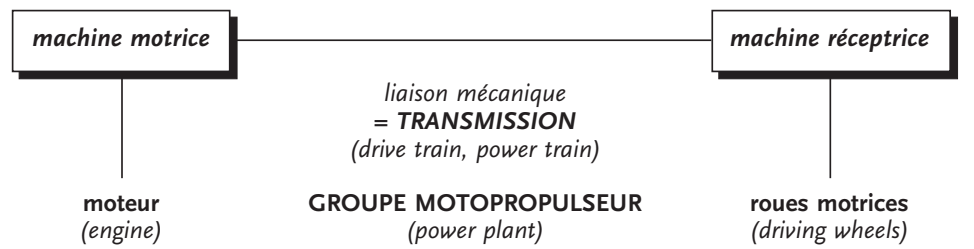

De là, on se transporterait dans des structures générique-spécifique présentant l'environnement logique de la notion de pompe et celle de moteur. Pour simplifier les choses, ce réseau arborescent serait scindé en deux parties, l'une d'elles faisant remonter jusqu'à la notion de machine dans la hiérarchie et l'autre illustrant l'éclatement de ces notions jusqu'à un niveau de spécificité raisonnable. (voir figure 6.)

L'arborescence particulière de la figure 5 est intéressante en raison de la symétrie remarquable qu'on y observe. Son intérêt réside en outre dans le fait que n'importe quelle machine, si l'on omet les machines de traitement ou de manipulation de l'information, a comme générique l'un des spécifiques s'y trouvant présentés.

FIGURE 5

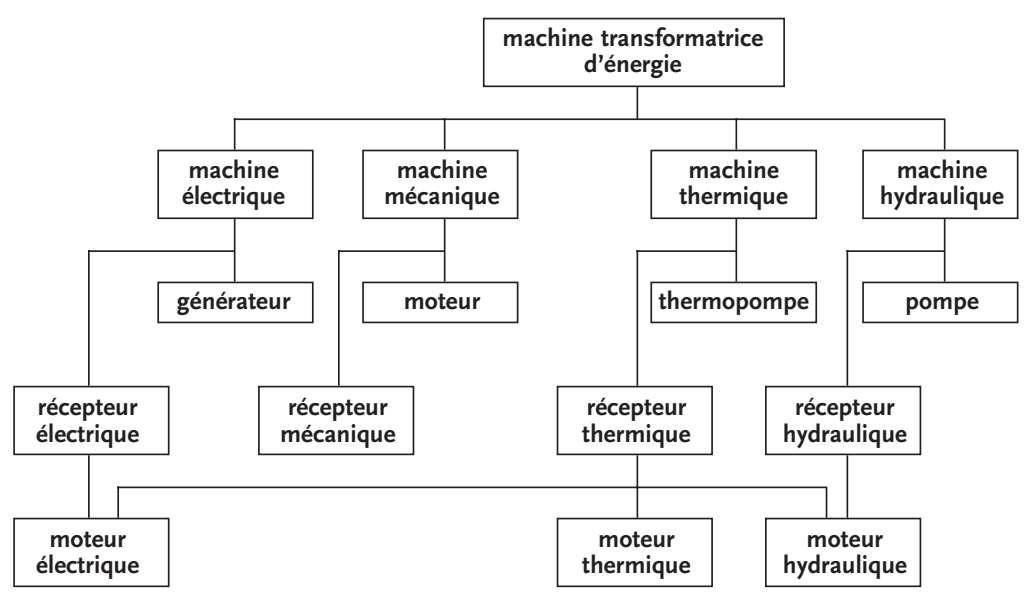

Ainsi, dans toute exploration d'une structure logique, il est essentiel de remonter toute l'arborescence, et de s'attarder aux notions qu'elle réunit, en raison notamment de l'importance du nombre de spécifiques qui se fondent sur elles, et pour lesquelles leur charge sémantique constitue un dénominateur commun. On peut voir là un phénomène de factorisation des connaissances, que l'on a intérêt à exploiter le plus systématiquement possible. Si l'on choisissait d'explorer le système notionnel des moteurs, notre prochain plan d'exploration serait le complément à la moitié supérieure de l'environnement logique représenté par la figure 6 ci-dessous, qui s'arrimerait à cette dernière par le nœud moteur et se poursuivrait vers le niveau référentiel par les différents niveaux de spécifiques en lesquels s'éclate la notion de moteur. 
FIGURE 6

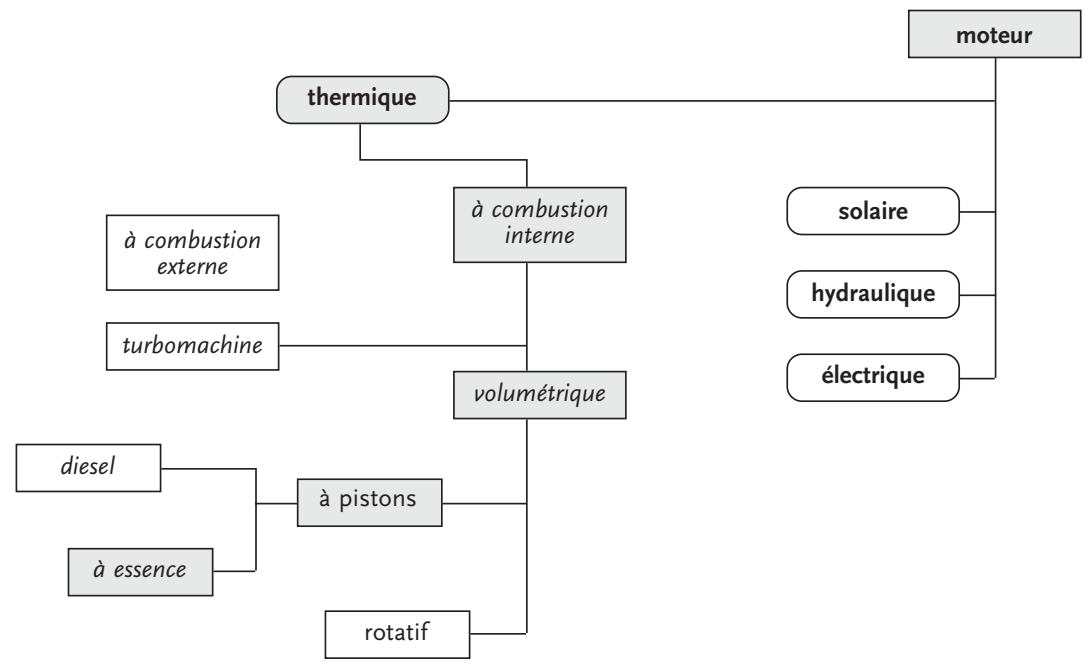

Une observation s'impose à propos de la navigation dans la structure logique que représentent les deux arborescences articulées autour de la notion moteur, la demi-arborescence inférieure pouvant être vue comme un gros plan du noud ciblé dans la demi-arborescence supérieure. Quand il s'agit de naviguer à l'intérieur d'un plan, la question se pose toujours de savoir à partir d'où, à partir de quel nœud ou notion. Et nous sommes alors «tiraillé» entre deux principes contradictoires. D'un côté, il est souhaitable d'aller du simple au complexe, et de l'autre du concret à l'abstrait. Le plus simple, c'est la racine de l'arborescence, mais c'est en même temps le plus abstrait. Une notion hypergénérique balaie large et filtre des objets individuels tout à fait hétéroclites, dont il est impossible de se faire une image.

À l'inverse, les notions les plus spécifiques filtrent des objets individuels formant des ensembles homogènes. Elles représentent des schémas de description riches, donc complexes, qui se rapprochent du niveau référentiel. On voit donc deux pôles. Il serait tentant de répondre que la solution se trouve entre les deux. Par ailleurs, un troisième principe s'applique: aller du connu vers le non-connu. Ce dernier critère nous aide à trancher. Il nous permet de comprendre que la bonne réponse ne se trouve pas dans le choix des niveaux centraux comme point de départ de l'exploration. La bonne réponse, c'est plutôt qu'il faut progresser à partir des deux pôles vers les zones centrales, en alternance.

\section{Les défis du formateur}

En résumé, la formation du traducteur spécialisé repose sur deux piliers: la traduction générale et l'initiation à la traduction spécialisée. Pour beaucoup, fonder l'initiation à la traduction spécialisée sur l'enseignement des langues de spécialité, c'est se placer sur un terrain glissant, tant les obstacles à surmonter pour bâtir un enseignement possédant quelque pertinence sont nombreux, tant l'investissement à faire pour y parvenir parait considérable, tant l'absence de tradition en cette matière rend les choses difficiles. 
On pourrait défendre l'idée que pour bien préparer les futurs traducteurs à la traduction spécialisée, il suffit de leur montrer comment mener efficacement une recherche documentaire et comment exploiter utilement un corpus pertinent. Nous avons pourtant le sentiment que l'acquisition par le traducteur d'une connaissance minimale de la langue de spécialité dans laquelle il est appelé à exercer sa compétence est un préalable incontournable. Personne n'aurait l'idée de traduire vers ou à partir d'une langue (générale) donnée sans commencer par apprendre la langue en question. Par ailleurs, faire reposer l'initiation à la traduction spécialisée sur l'apprentissage des langues de spécialité, c'est un moyen de sortir d'une forme d'enseignement fondée sur une pédagogie punitive.

Si enseigner la traduction générale revient à communiquer un savoir-faire, dispenser un enseignement visant l'acquisition des langues de spécialité se ramènerait plutôt à communiquer une manière d'apprendre. Pour être plus précis, il s'agit d'apprendre à reconnaître quoi apprendre, et d'apprendre comment apprendre. L'enseignement des langues de spécialité impose de concevoir et de proposer une pédagogie ou un modèle d'apprentissage, nécessairement original, compte tenu à la fois du manque de tradition et de la nature très particulière des connaissances ciblées.

Notre modèle d'apprentissage, qui est une sorte de cadre pédagogique, serait utilisable aussi bien pour bâtir un cours que pour mener une démarche d'autoapprentissage. Les ingrédients qu'il réunit sont variés et complémentaires. On y a fait une place à la métaconnaissance, sans doute plus utile pour l'enseignant (et qui lui sert à définir une démarche optimale d'apprentissage et un contenu pertinent) que pour l'apprenant, mais à voir néanmoins comme un levier général. D'autres ingrédients s'apparentent à des stratégies, dans la mesure où leur objet est avant tout d'aider à bien cibler les connaissances spécialisées (à distinguer des connaissances du spécialiste) dont l'acquisition est essentielle. D'autres encore sont des principes, sur lesquels on s'appuie pour définir un ordre de présentation ou d'exploration des notions. D'autres, enfin, sont des leviers concrets comme les groupements et les représentations des notions sous des formes originales, qui servent de base pour l'élaboration d'un discours sur les langues de spécialité centré sur les connaissances essentielles à acquérir.

Nous avons présenté une défense, sous la forme d'une argumentation logique, d'un type d'enseignement dont on peut penser qu'il n'est pas viable, compte tenu des paramètres définissant la situation d'apprentissage. Les obstacles sont examinés, et pour chacun d'eux nous proposons une façon de le surmonter.

Il existe cependant une argumentation concrète, que nous n'avons pas invoquée jusque-là, et qui mériterait de l'être. Elle s'articule autour de trois constats. Le premier, c'est que les connaissances classiques et essentielles sur les langues de spécialité mises de l'avant tout au long de notre argumentation existent bien dans la mémoire des traducteurs spécialisés possédant une compétence reconnue. Le deuxième constat est le fait que ces connaissances centrales, s'apparentant notamment aux brouillages interlinguistiques, ne se trouvent encodées nulle part. Quant au troisième constat, c'est le fait que nos étudiants ont une capacité et une soif de s'approprier des connaissances pertinentes absolument considérables, et que nous sommes incapables de nous placer à la hauteur de leurs attentes, pour toutes sortes de raisons, bonnes ou mauvaises.

Ces constats appellent une réponse. La nôtre donne seulement une direction. Nous nous sommes contenté de mettre de l'avant une manière d'enseigner une matière 
très particulière, qui est aussi une manière d'apprendre, reposant sur l'adoption de stratégies, sur la recherche et l'utilisation de leviers, et sur l'application de principes. Il s'agit d'une réponse abstraite, et si l'on adhère à cette conception de l'enseignement des langues de spécialité, tout reste à faire, en particulier pour déterminer les contenus pertinents, pour aller chercher les connaissances classiques évoquées et les mettre dans la forme que nous suggérons. Tout reste à faire aussi pour mettre en place les modalités pratiques d'utilisation d'un tel modèle.

Ainsi, la première question qui se pose est comment, de façon pratique, élaborer un tel enseignement selon le modèle présenté? Ce sont sans doute les exigences représentées par une telle tâche qui expliquent que l'on se soit généralement détourné de cette forme d'enseignement. Ce n'est pas toujours le fait qu'on ne reconnaisse pas sa pertinence. Simplement pour être en mesure de définir des contenus pertinents à l'intérieur d'un grand domaine, il faut posséder une très bonne vue d'ensemble du domaine. Pour extraire les connaissances essentielles, les articuler et les inscrire dans un discours centré sur la langue, il faut du temps, beaucoup de temps, et une bonne dose de patience et de détermination.

La cause n'est pas pour autant désespérée. Quoi qu'on dise, de toute façon, le professeur de traduction spécialisée devra toujours faire des efforts pour s'approprier les connaissances essentielles sur les langues de spécialité dans les domaines qu'il a investis, même si ce n'est pas sous une forme susceptible de donner lieu à un enseignement structuré, et il devra constamment améliorer sa connaissance de l'organisation des domaines en question. Par ailleurs, jamais la matière première à partir de laquelle un enseignement des langues de spécialité serait susceptible de s'élaborer n'aura été plus accessible. Jamais les outils de gestion de cette matière première (nous pensons aux bases de données) n'auront été plus performants et conviviaux. Et jamais la mise en forme de produits finis n'aura été autant simplifiée grâce à toute une panoplie d'outils de présentation informatiques. 